\title{
Study on detection method for mixed network structure robustness in 4G network
}

\author{
Chen Hui-min \\ (Guangdong Vocational College of Post and Telecom, Guangzhou 510630,China)
}

Keywords: BP neural network; robustness; 4G network;

\begin{abstract}
G}$ network is researched. BP neural network algorithm is utilized to detect mixed network structure robustness in 4G network, because the $4 \mathrm{G}$ network reflects the exchange of information between individual and individual, randomness factors of network information is prominent, weights of BP neural network transmit into solid condition, leading to large detection error for robustness. Therefore, this paper proposes a fuzzy correlation theory to construct the detection model for mixed network structure robustness in $4 \mathrm{G}$ network. On the basis of given new robustness measure for mixed network structure in 4G network, according to small domain communication efficiency and the size of connected graph, the two attribute are utilized to calculate the robustness of single node of mixed network structure in 4G network, the quantized robust value is divided into two independent feature subspace and non feature subspace, in the corresponding space, the correlation robustness of region nodes is calculated, so as to complete robustness detection of mixed network structure in $4 \mathrm{G}$ network. Simulation results show that, the new $4 \mathrm{G}$ network mixed network structure robustness detection model can reduce evaluation error of mixed network structure in 4G network greatly, the system has better robustness, can effectively promote the operation and development of $4 \mathrm{G}$ network.
\end{abstract}

\section{Introduction}

The 4G network model is the communication platform for communicating between individuals, because of the openness of $4 \mathrm{G}$ network, the structure of $4 \mathrm{G}$ network is complicated, the network structure crisis and network structure safety problems exist commonly [1]. The network structure robustness analysis and the trustiness mathematical model of $4 \mathrm{G}$ network are studied, to establish 4G network structure robustness detection mechanism [2-4], so as to promote the healthy development and operation of $4 \mathrm{G}$ network mode, and protect scientific development of application field like 4G network user interests and security, which have great research value and practical value [5-7].

BP neural network algorithm is utilized to detect mixed network structure robustness in 4G network, because the $4 \mathrm{G}$ network reflects the exchange of information between individual and individual, randomness factors of network information is prominent[8-10], weights of BP neural network transmit into solid condition, leading to large detection error for robustness.

In order to avoid the disadvantages of the traditional algorithm, this paper proposes a fuzzy correlation theory to construct the detection model for mixed network structure robustness in 4G network. On the basis of given new robustness measure for mixed network structure in 4G network, according to small domain communication efficiency and the size of connected graph, the two attribute are utilized to calculate the robustness of single node of mixed network structure in 4G network, the quantized robust value is divided into two independent feature subspace and non-feature subspace, in the corresponding space, the correlation robustness of region nodes is calculated, so as to complete robustness detection of mixed network structure in 4G network. Simulation results show that, the new $4 G$ network mixed network structure robustness detection model can reduce evaluation error of mixed network structure in $4 \mathrm{G}$ network greatly, the system has better robustness, can effectively promote the operation and development of $4 \mathrm{G}$ network. 


\section{2 principle of mixed network structure robustness detection in $4 \mathrm{G}$ network based on the related nodes}

2.1 The quantitative calculation of robustness of single node in mixed network structure of $4 \mathrm{G}$ network

Through communication efficiency of the mixed network structure in 4G network and other node robustness features and the following formula, the network node robustness of $4 \mathrm{G}$ network structure is obtained:

$$
S=\sqrt{\left(t_{1}-p_{1}\right)^{2} w_{1}+\left(t_{2}-p_{2}\right)^{2} w_{2}+\ldots . . .\left(t_{14}-p_{14}\right)^{2} w_{14}}
$$

In the formula, $t_{i}$ represents the robustness feature vector of mixed network structure nodes in $4 \mathrm{G}$ network requires to be detected, $p_{i}$ represents standard nodes feature vector of $4 \mathrm{G}$ network; $w_{i}$ is weight.

The above formula does not describe the fluctuation of $4 \mathrm{G}$ network node robustness features and the relevance of robustness and testing process. After 4G network nodes are affected adversely, its robustness will be reduced, but model constructed by actual sampling has strong stability. Therefore, the above formula results in certain error for sampling nodes robustness characteristics values. Thus the fluctuation of node robustness features and the relevance of robustness and testing process have to be analyzed comprehensively, robustness feature attributes can be adjusted reasonably, and the fluctuation of node robustness features can be analyzed sufficiently when computing similarity. Robustness characteristic quantity of original node in network structure of $4 \mathrm{G}$ networks are $I_{1}$ and $I_{2}$ respectively, using $\Delta=I_{1}-I_{2}$ to describe fluctuations of robustness node feature vector, in accordance with these variables, the computing formula of node similarity can be accessed:

$$
S\left(I_{1}, I_{2}\right)=P\left(\Delta \in \Omega_{i}\right)=P\left(\Omega_{i} \mid \Delta\right)
$$

In the formula, $\Omega_{i}$ represents variation quantity of detection model of a node in mixed network structure of $4 \mathrm{G}$ network under different influencing environment, namely the similar variation; $\Omega_{E}$ is used to describe variation of robustness testing model, under another interference environment, namely the heterogeneous variation. The $P\left(\Omega_{i} \mid \Delta\right)$ is used to describe similar posteriori probability when the robustness is fluctuating, the probability can be obtained with $P\left(\Delta \mid \Omega_{i}\right)$ and $P\left(\Delta \mid \Omega_{E}\right)$. Therefore, the optimized similarity arithmetic can analyze the situation of node robustness affected adversely comprehensively, such as the relevance between testing error caused by the fluctuation of node robustness and the change. The optimized computation formula of robustness is as shown in the following formula:

$$
S\left(I_{1}, I_{2}\right)=\frac{P\left(\Delta \mid \Omega_{i}\right) P\left(\Omega_{i}\right)}{P\left(\Delta \mid \Omega_{i}\right) P\left(\Omega_{i}\right)+P\left(\Delta \mid \Omega_{E}\right) P\left(\Omega_{E}\right)}
$$

In the formula, $P\left(\Omega_{E}\right)$ is used to describe the specific external conditions. Such as the quantitative indicators of adverse influencing factors under detection conditions, and the association probability of number of tests. Thus, robustness detection process can be transformed into a process of calculating related probability, calculates the probability of node robustness characteristic change $\Delta$ belongs to $\Omega_{i}$ or $\Omega_{E}$, so as to make accurate analysis for node robustness of mixed network structure in $4 \mathrm{G}$ network.

2.2 calculation of similarity based on correlation degree of

By adopting the above analysis method to process quantization operation for nodes in mixed network structure of $4 \mathrm{G}$ network, and the corresponding results are processed as follows:

The calculated single node robustness is divided into two sub regions without any relevance: 


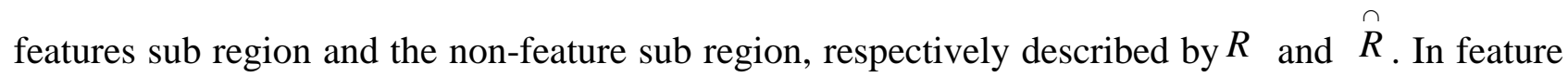
sub region $R, \quad$ M node robustness feature vector similarity node of low latitude are as shown in the following formula:

$$
\hat{P}(\Delta \mid \Omega)=\left[\frac{\exp \left(-\frac{1}{2} \sum_{i=1}^{M} \frac{y_{i}^{2}}{\lambda_{i}}\right)}{(2 \pi)^{M / 2} \prod_{i=1}^{M} \lambda_{i}^{1 / 2}}\right] \cdot\left[\frac{\exp \left(-\frac{\varepsilon^{2}(\Delta)}{2 \rho}\right)}{(2 \pi \rho)^{\frac{(N-M)}{2}}}\right]-P_{F}(\Delta \mid \Omega) \bullet P_{F}(\Delta \mid \Omega)
$$

Wherein, $P_{F}(\Delta \mid \Omega)$ represents the actual edge density of R sub region; $\stackrel{\Lambda}{P_{F}}(\Delta \mid \Omega)$ is edge density predicted in $\stackrel{\hat{R}}{;} y_{i}$ represents the principal component; $\varepsilon^{2}(\Delta)$ is residual energy; the average value of eigenvalues of sub region $\stackrel{\cap}{R}$ can be used to describe the weight parameters $\rho$ :

$$
\rho=\frac{1}{N-M} \sum_{i=M+1}^{N} \lambda_{i}
$$

Among them, $P\left(\Delta \mid \Omega_{i}\right)$ and $P\left(\Delta \mid \Omega_{E}\right)$ are subject to the three-dimensional Gauss distribution, and:

$$
\left\{\begin{array}{l}
P\left(\Delta \mid \Omega_{E}\right)=\frac{e^{-1 / 2} \Delta^{r} \sum E^{-1} \Delta}{(2 \pi)^{\frac{D}{2}}\left|\sum E\right|^{1 / 2}} \\
P\left(\Delta \mid \Omega_{i}\right)=\frac{e^{-1 / 2} \Delta^{r} \sum E I^{-1} \Delta}{(2 \pi)^{\frac{D}{2}}\left|\sum I\right|^{1 / 2}}
\end{array}\right.
$$

Among them, $\sum$ is used to describe the covariance matrix. The difference between $I_{k}$ and $I_{j}$ are obtained, the difference is $\Delta$ vector, $P\left(\Delta \mid \Omega_{i}\right)$ and $P\left(\Delta \mid \Omega_{E}\right)$ are calculated from the feature vector of principal components of Gauss density function within class and between class, then computing correlation robustness of mixed network structure of $4 \mathrm{G}$ network. In order to reduce the complexity of the algorithm, two albino vectors can be fit into robustness results:

$i_{j}=\wedge_{I}^{-1 / 2} \vee_{I} I_{j}, \quad e_{j}=\wedge_{E}^{-1 / 2} \vee_{E} I_{j}$

In the formula, $\wedge$ and $\vee$ respectively represents the diagonal matrix of the maximum robustness eigenvalues and eigenvectors matrix corresponding to these eigenvalues of $\sum I$ and $\sum E$, dimension of corresponding sub regions are $M_{I}$ and $M_{E}$. The above probability calculation process can be regarded as the process to calculate robustness of mixed network structure in overall $4 \mathrm{G}$ network, there are:

$$
\left\{\begin{array}{l}
P\left(\Delta \mid \Omega_{I}\right)=\frac{e^{-1 / 2} \Delta^{T} \sum I^{-1} \Delta}{(2 \pi)^{D / 2}\left|\sum I\right|^{1 / 2}} \\
P\left(\Delta \mid \Omega_{E}\right)=\frac{e^{-1 / 2} \Delta^{T} \sum E^{-1} \Delta}{(2 \pi)^{D / 2}\left|\sum E\right|^{1 / 2}}
\end{array}\right.
$$

\section{3 simulation experiments and results analysis}

The simulation experiment is carried out in the Matlab simulation software, through the long-term data collected by the research group in 4G network environment, 10000 data were 
selected from 4G network mixed data network structure as the training data, 2205 data were regarded as test data, the data was normalized, so as to improve the convergence speed and the robustness properties of mixed network structure. The data is input into the detection system for the robustness of mixed network structure in $4 \mathrm{G}$ network, the obtained training output results as shown in the diagram below, it can be seen from Figure 1, using this system to train the network, after 23 times of training, the preset precision 0.05 can be achieved.

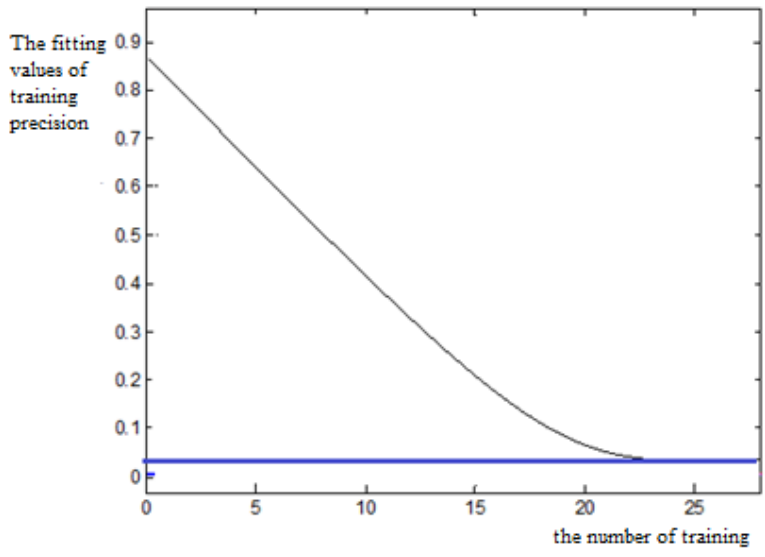

Figure 1 simulation results of the number of training times and the training precision

Eight groups of training samples were selected, the expected output and actual output, that is observation value and fitting value, were shown in Table1. 5 groups of test ample simulation were chosen, observation value and fitting value were shown in Table2, It can be known from Table2, that the fitting value between expected output value and actual output value has small error, the system has better training effect, and strong robustness.

Table 1 the observation value and the fitted value of training samples

\begin{tabular}{|c|c|c|c|c|c|c|c|c|}
\hline Training Sample & 1 & 2 & 3 & 4 & 5 & 6 & 7 & 8 \\
\hline The observed value & 3.7 & 3.2 & 2.9 & 3.4 & 3.6 & 3.9 & 2.1 & 1.9 \\
\hline The fitted value & 3.34 & 2.95 & 2.64 & 3.17 & 3.47 & 3.98 & 2.18 & 1.87 \\
\hline \multicolumn{9}{|c|}{ Table 2 the observation value and the fitted value of test samples } \\
\hline Training Sample & & & 2 & & 3 & 4 & & 5 \\
\hline The observed value & & & 3 & & 3.43 & 3 & & 3 \\
\hline The fitted value & & & 2.947 & & 2.776 & 3.198 & & 3.125 \\
\hline
\end{tabular}

\section{Conclusion}

During the detection process of mixed network structure robustness in 4G network, aiming at the defect caused by prominent randomness factors of 4G network information, weights of BP neural network transmitted into curing condition, and large detection error for robustness, this paper proposes a fuzzy correlation theory to construct the detection model for mixed network structure robustness in 4G network. On the basis of given new robustness measure for mixed network structure in 4G network, according to small domain communication efficiency and the size of connected graph, the two attribute are utilized to calculate the robustness of single node of mixed network structure in $4 \mathrm{G}$ network, the quantized robust value is divided into two independent feature subspace and non-feature subspace, in the corresponding space, the correlation robustness of region nodes is calculated, so as to complete robustness detection of mixed network structure in 4G network. Simulation results show that, the new $4 \mathrm{G}$ network mixed network structure robustness detection model can reduce evaluation error of mixed network structure in 4G network greatly, the system has better robustness, can effectively promote the operation and development of 4G network. 


\section{References}

[1] Li Xiangming. 4G communication network architecture and its key technology analysis [J]. Science, 2013.4:159-160.

[2] Yu Xiaonan. 4G communication network architecture and its key technology analysis [J]. Value engineering, 2012.179-180.

[3] Liu Chunyan. FTTH network structure analysis [J]. Study on optical communications, 2005.4:30-32.

[4] Yao Qiling, Yue Jun, Sui Yanfeng, Quan Xiao, Bai Yan, Qi Jingli. Assessment method for LTE network structure based on MR data [J]. Mobile communication, 2013.21:27-31.

[5] Chen Bing, Wang Lisong. Network topology discovery based on three tier architecture [J], the application of computer, 2002.6:23-25.

[6] Li Zhaolei, Zhang Yaqi. Analysis of the network organization structure of modern logistics enterprises [J]. Journal of Hebei Jiaotong Vocational and Technical College, 2008.1:7-9.

[7] Qiao Jinsuo, Wang Xifu, Shen Xisheng, Shi Liang. Robustness evaluation and application of coal transport network structure [J]. Journal of transportation systems engineering and information, 2013.4:126-133.

[8] Du Wei, Cai Meng, Du Haifeng. Application study on robust indicators of network structure [J]. Journal of Xi'an Jiao Tong University, 2010.4:93-97.

[9] Yang Juan, Yan Biao, Chen Wanpei. The new technology of 4G and its comparison with 3G [J]. Information technology, 2004.98-101

[10] Liu Wei, Ding Zhijie. The research progress and key technology of 4G mobile communication system [J]. China data communication.2004.8-12 